\title{
Profiling ascidian promoters as the primordial type of vertebrate promoter
}

\author{
Kohji Okamura ${ }^{1,2}$, Riu Yamashita', Noriko Takimoto ${ }^{3}$, Koki Nishitsuji ${ }^{3,4}$, Yutaka Suzuki ${ }^{5}$, Takehiro G Kusakabe ${ }^{4}$, \\ Kenta Nakai ${ }^{*}$ \\ From Asia Pacific Bioinformatics Network (APBioNet) Tenth International Conference on Bioinformatics - First \\ ISCB Asia Joint Conference 2011 (InCoB/ISCB-Asia 2011) \\ Kuala Lumpur, Malaysia. 30 November - 2 December 2011
}

\begin{abstract}
Background: CpG islands are observed in mammals and other vertebrates, generally escape DNA methylation, and tend to occur in the promoters of widely expressed genes. Another class of promoter has lower $\mathrm{G}+\mathrm{C}$ and $\mathrm{CpG}$ contents, and is thought to be involved in the spatiotemporal regulation of gene expression. Non-vertebrate deuterostomes are reported to have a single class of promoter with high-frequency CpG dinucleotides, suggesting that this is the original type of promoter. However, the limited annotation of these genes has impeded the largescale analysis of their promoters.

Results: To determine the origins of the two classes of vertebrate promoters, we chose Ciona intestinalis, an invertebrate that is evolutionarily close to the vertebrates, and identified its transcription start sites genome-wide using a next-generation sequencer. We indeed observed a high CpG content around the transcription start sites, but their levels in the promoters and background sequences differed much less than in mammals. The CpG-rich stretches were also fairly restricted, so they appeared more similar to mammalian CpG-poor promoters.

Conclusions: From these data, we infer that CpG islands are not sufficiently ancient to be found in invertebrates. They probably appeared early in vertebrate evolution via some active mechanism and have since been maintained as part of vertebrate promoters.
\end{abstract}

\section{Background}

Among the 16 DNA dinucleotides, the CpG dinucleotide is unique in terms of its frequency in genomic sequences. This most probably results from the DNA methylation system because the DNMT1 and DNMT3 families of the deuterostomes, such as echinoderms and chordates, predominantly target the 5 position of cytosine residues only in the CpG dinucleotide [1]. Because the deamination of 5-methylcytosine is not recognized by the DNA repair mechanisms, $\mathrm{CpG}$ is rapidly mutated to TpG or to its complementary dinucleotide CpA [2]. Therefore, deuterostome organisms, except for Oikopleura dioica [3], display a globally reduced frequency of

\footnotetext{
* Correspondence: knakai@ims.u-tokyo.ac.jp

'Human Genome Centre, Institute of Medical Science, University of Tokyo,

Tokyo, Japan

Full list of author information is available at the end of the article
}

the CpG dinucleotide compared with its expected frequency calculated from actual numbers of guanine and cytosine residues $[4,5]$. Interestingly, they also display skewed distributions of the $\mathrm{CpG}$ dinucleotide across their genomes, so that their genomes contain CpG-poor and CpG-rich domains [6,7]. In amphibians, avians, and mammals, the CpG-rich domains are much shorter than the $\mathrm{CpG}$-poor domains and are generally known as CpG islands [8].

CpG islands are good markers of some classes of genes because they are often linked to the promoters of those genes [9]. In most cases, CpG islands escape DNA methylation, which suppresses gene expression in general, in almost every tissue [10] and function as part of the gene promoter [11]. Hence, $\mathrm{CpG}$ islands tend to be related to ubiquitously or broadly expressed genes, whereas promoters that lack a $\mathrm{CpG}$ island are involved 
in the spatiotemporal regulation of the genes [12]. It is important to note that mammalian promoters can be thus divided into the two distinct classes, not only structurally but also functionally. In the human genome, CpG-rich promoters or $\mathrm{CpG}$ island promoters are dominant, occurring more than twice as often as CpG-poor promoters $[13,14]$.

As anticipated for a vertebrate taxon, CpG island promoters were indeed experimentally identified in fish by an analysis of transcription start sites (TSSs) [15]. The presence of two classes of promoters in fish, amphibians, reptiles, avians, and mammals has since been confirmed in silico [16]. In that study, the authors analysed the distributions of the normalized CpG contents (the ratio of the observed $\mathrm{CpG}$ number to the expected $\mathrm{CpG}$ number, called the "CpG score" hereunder) of the promoter sequences in six vertebrate genomes and showed bimodal distributions for all of them. Furthermore, the structural bimodality was shown to correspond to functionally distinct classes of genes. The authors also analysed three invertebrate promoters, of one sea urchin and two ascidian (sea squirt) species, and found unimodal distributions of high CpG scores, unlike the distributions observed in the vertebrate promoters. This led them to propose that the vertebrate promoter classes differentiated at an early stage of vertebrate evolution, with global DNA methylation and subsequent deamination. This is basically consistent with the formerly accepted evolutionary hypothesis of CpG islands $[17,18]$.

If this hypothesis is true, do the non-vertebrate deuterostomes (e.g. echinoderms, lancelets, and ascidians) have $\mathrm{CpG}$ islands in their genomes? Currently, the presence of $\mathrm{CpG}$ islands in invertebrate animals is unclear. It is possible to apply any criteria that define a $\mathrm{CpG}$ island to their genomic sequences and identify some islands. Nevertheless, we were interested in determining whether there are CpG island-like sequences in invertebrate genomes that are associated with transcription initiation, and how and when these sequences appeared during evolution.

To address this issue, we identified the TSSs of Ciona intestinalis by a combination of the oligo-capping method [19] and massive-scale cDNA sequencing (RNA-seq, specifically TSS-seq) [20]. The widely used model organism $C$. intestinalis is an ascidian tunicate, which although an invertebrate, is most closely related to the vertebrates [21]. Although the ascidian evolved from the last common ancestor of the ascidians and vertebrates, it can be presumed to retain many more features of the ancestral organism than do extant vertebrates. It is well known that the enrichment of the CpG dinucleotides in CpG island promoters is maximum in TSSs $[12,13]$, so TSSs constitute candidate regions in which $\mathrm{CpG}$ island promoters or $\mathrm{CpG}$ island- like sequences might occur in the invertebrate genome. Incidentally, this approach that targets TSSs also circumvents the confusion arising from CpG-rich sequences that are indifferent to transcription initiation. In the computational study mentioned above, promoter regions were defined using the RefSeq database, which is a curated collection of publicly available nucleotide sequences [16]. It is likely that many of the cDNA entries are truncated or incomplete at the 5 ' end which makes the definition of their promoter regions unreliable. More importantly, the TSSs of approximately half of all ascidian genes can hardly be determined because of mRNA 5'-leader trans-splicing [22-24]. The 5' ends of those primary transcripts, termed the outron, are discarded via the trans-splicing reaction. This fact is easily exemplified by downstream operonic genes, which are resolved from their primary transcripts by trans-splicing [25]. Although it is almost impossible to know TSSs of them, it is essential to be distinguished from non-transspliced genes and to know the most 5' end position of the processed transcripts. Analyzing these data, we determined the structural features of the ascidian promoters and compared them with human promoters to identify and characterize their similarities and differences. To extend our understanding of gene regulation in higher eukaryotes, we undertook to clarify the origin of CpG islands and the two classes of vertebrate promoters.

\section{Results}

In this study, we chose $C$. intestinalis embryos at the mid-tailbud stage (Additional file 1: Figure S1) for the genome-wide identification of TSSs. Since whole embryos still retaining the notochord contain a wide range of cell types, we may cover a large part of ascidian promoters. Total RNA was extracted from embryos and was subjected to oligo capping in which the $5^{\prime}$ cap of the mRNA was replaced with a synthetic RNA oligonucleotide (see Methods). After cDNA synthesis and subsequent PCR, we undertook massively parallel sequencing using the Illumina Genome Analyzer. We obtained two data sets containing fragments of different lengths $36 \mathrm{nt}$ or $48 \mathrm{nt}$. Because we read the sequences from the 3' end of the RNA oligonucleotide, all the sequences obtained should start with GG at their $5^{\prime}$ ends (see Methods). We recovered only the reads that started with GG, but then trimmed the GG from those. Although the genic sequences were trimmed by two nucleotides, this protocol eliminated dubious sequences that do not start with the dinucleotide. We also eliminated sequences containing undetermined nucleotides other than T, C, A, and G, yielding 4,247,902 reads of $34 \mathrm{nt}$ and 4,770,608 reads of $46 \mathrm{nt}$. To detect the spliced leader (SL) of $C$. intestinalis, we considered, in addition 
to the canonical 16-nt sequence, all similar sequences, allowing a 1-nt mismatch or indel and some previously reported variants [24]. The 34-nt data set consisted of 1,849,849 non-trans-spliced and 2,398,053 trans-spliced reads and the 46-nt data set consisted of 2,052,230 nontrans-spliced and 2,718,378 trans-spliced reads. Even if some SL-related 5' mRNA sequences escaped from being detected by this process, it is unlikely such reads would map to the genome in the following step. Mapping or alignment to the $\mathrm{KH}$ assembly [25] was performed as described in the Methods. Sequences that mapped to more than one locus (multiple hits) were not considered further. The numbers of mapped 34-nt and 46-nt reads were 1,017,283 (non-trans-spliced), 1,932,570 (trans-spliced), 939,092 (non-trans-spliced), and 1,237,720 (trans-spliced), respectively. Because the original 5'-segment of a pre-mRNA is discarded during the trans-splicing reaction, mature trans-spliced mRNAs do not contain the initial segment of the primary transcript and therefore lack the information required to precisely identify TSS [22]. Therefore, we decided to mainly examine non-trans-spliced reads to provide valid data for the promoter analyses presented here. The genomic positions to which the 5 ' ends of the reads were aligned were defined as TSSs. The read counts were converted to values in parts per million ( $\mathrm{ppm}$ ) for transcript abundance estimation and normalization, and both of the short and long data sets were merged. The TSSs, which are generally scattered around a promoter region [26], were then clustered into 100-bp bins to define each promoter. In other words, two reads located more than $100 \mathrm{bp}$ apart without any other reads between them were considered to be regulated by two separate promoters [26]. In this clustering process, TSSs represented by reads occurring at less than $0.5 \mathrm{ppm}$ were not considered. However, once promoters were defined, all the TSSs in the bins were counted to estimate the abundance of transcripts from each cluster. Because we can assume that every cell contains approximately one million mRNA molecules, we can consider the values in ppm as copy numbers of the transcripts in a cell [27]. We set a threshold of $1.0 \mathrm{ppm}$ to exclude transcriptional noise. As a result, we obtained 6312 and 8753 promoters for non-trans-spliced and trans-spliced genes, respectively, that could be considered active in the tailbud embryos. The most frequent TSS in each promoter (and if there were several, the most upstream one) was selected as its representative TSS. If the corresponding genes were found in the $\mathrm{KH}$ gene model [25], the gene names were also tabulated (Additional file 2: Tables S1 and S2). Note that one gene can have several alternative promoters.

The initiator (Inr) motif, which spans the TSS, is the most commonly occurring sequence motif observed in metazoans [28]. Its consensus sequence between mammals and fruit fly is pyrimidine-purine (YR), where $R$ corresponds to the exact TSS [29]. By aligning core promoter sequences of all the 6312 non-trans-spliced transcripts with consideration of their orientation, we confirmed that the ascidian promoters also follow the YR consensus, suggesting that the sequence processes described above are plausible (Figure 1). In this figure, all the representative TSSs are aligned at position 0 . The next positions upstream and downstream are designated -1 and +1 , respectively. This notation is used in the rest of the present paper. Another alignment of all the 8753 trans-spliced transcripts is also shown. In this case, however, the position 0 means the most 5 ' end of the transcripts after removing SLs.

We then examined the genome-wide distributions of the CpG scores in both the whole genome and the promoters of non-trans-spliced transcripts, using a sliding window of $1 \mathrm{~kb}$. To compare them with the corresponding vertebrate distributions, we performed the same analysis using the human genome (Figure 2). We defined a sequence fragment from -499 to +500 as a promoter. A similar analysis of the CpG-score distributions has already been reported [16]. Although the definitions of the promoter sequences differ in these studies, we obtained fundamentally identical results. The human genome is globally methylated and CpG dinucleotides occur in bulk at only one-fifth of the expected frequency [17]. In contrast, the ascidian genome contains approximately equal amounts of methylated and unmethylated regions, which may have resulted in CpG-poor and CpG-rich sequences, respectively $[7,14]$. Intriguingly, the ascidian and human promoters show unimodal and bimodal distributions, respectively. The latter distribution indicates that the human has two classes of promoters, CpG-poor and CpG-rich. The CpG-rich promoters can be considered to contain a CpG island. In contrast, the ascidian promoters generally tend to have high $\mathrm{CpG}$ scores and exhibit a unimodal distribution. This observation led to the hypothesis that human CpG-poor promoters emerged with the deamination of methylated CpG dinucleotides in CpG island promoters [16]. Using our experimental data, we intended to substantiate this idea and define the $\mathrm{CpG}$ islands in the invertebrate genome.

We excised 4-kb promoter sequences (2 kb upstream and $2 \mathrm{~kb}$ downstream from each representative TSS) of the ascidian non-trans-spliced, and human CpG-poor, and CpG-rich promoters, and aligned them with consideration of the transcriptional orientation to determine the overall changes in the $\mathrm{CpG}$ scores and $\mathrm{G}+\mathrm{C}$ contents in the vicinity of the TSSs (Figure 3). We used Database of Transcription Start Sites (DBTSS) to select the human promoter sequences [27]. The methodological details 
such as grouping human CpG-poor and CpG-rich promoters are described in the Methods. Our results confirmed that the ascidian promoters tended to have high CpG score and $\mathrm{G}+\mathrm{C}$ contents around TSS, as was observed in the human promoters. However, judging from the heights and extents (widths) of the peaks around the TSSs, the ascidian promoters seem more similar to the human CpG-poor promoters than to the human $\mathrm{CpG}$ island promoters (Figure 3B). Although the ascidian TSSs exhibited quite high CpG score, this fact does not necessarily mean that they have high frequency of the $\mathrm{CpG}$ dinucleotide (Figure $3 \mathrm{~A}$ ). The low content of $\mathrm{G}+\mathrm{C}$ underestimated the expected number of $\mathrm{CpG}$, which in result increased the ratio of the observed over expected numbers of the dinucleotide, i.e. CpG score. Hence, we defined "CpG content" to show its plain density (see Methods) and drew the changes (Figure 3C). The heights and extents were comparable between the ascidian and CpG-poor promoters and their contents were regularly lower than the expected content for any dinucleotide, 0.0625 or $1 / 16$. In addition to $\mathrm{CpG}$, we also analysed the changes in all the other dinucleotide scores in the vicinity of the TSSs (Additional file 3: Figure S2). Distinct features were also observed at the TSSs for all these dinucleotide scores. This information may possibly be used to predict the locations of promoters and their corresponding genes.

Among the dinucleotides, the local frequencies of TpG and $\mathrm{CpA}$ can be used as indicators of DNA methylation levels [4]. We calculated the TpG and CpA scores for 1$\mathrm{kb}$ promoter sequences and charted their distributions for the three classes of promoters (Figure 4). All the six histograms showed a unimodal bell-shaped distribution, e.g. $p<10^{-15}$ by Kolmogorov-Smirnov test for Figure 4A, indicating that they were formed by promoters having homogeneous characters in terms of the dinucleotide scores. Whereas the distributions of the human CpG island promoters are centered at the value of 1.0, the distributions of the ascidian and CpG-poor promoters are shifted to higher-score regions, where observed numbers of the deaminated dinucleotides are larger than their expected numbers. It is more likely that deamination of CpG sites are common. The high frequency of deamination in the ascidian and CpG-poor promoters suggests that these regions are relatively methylated unlike $\mathrm{CpG}$ islands. Because mutations in somatic cells have not been transmitted evolutionarily, what we observed here is the result occurred in germ line. The DNA methylation could be tissue-, stage-, or cell-type-specific and play a role in spatiotemporal gene regulation.

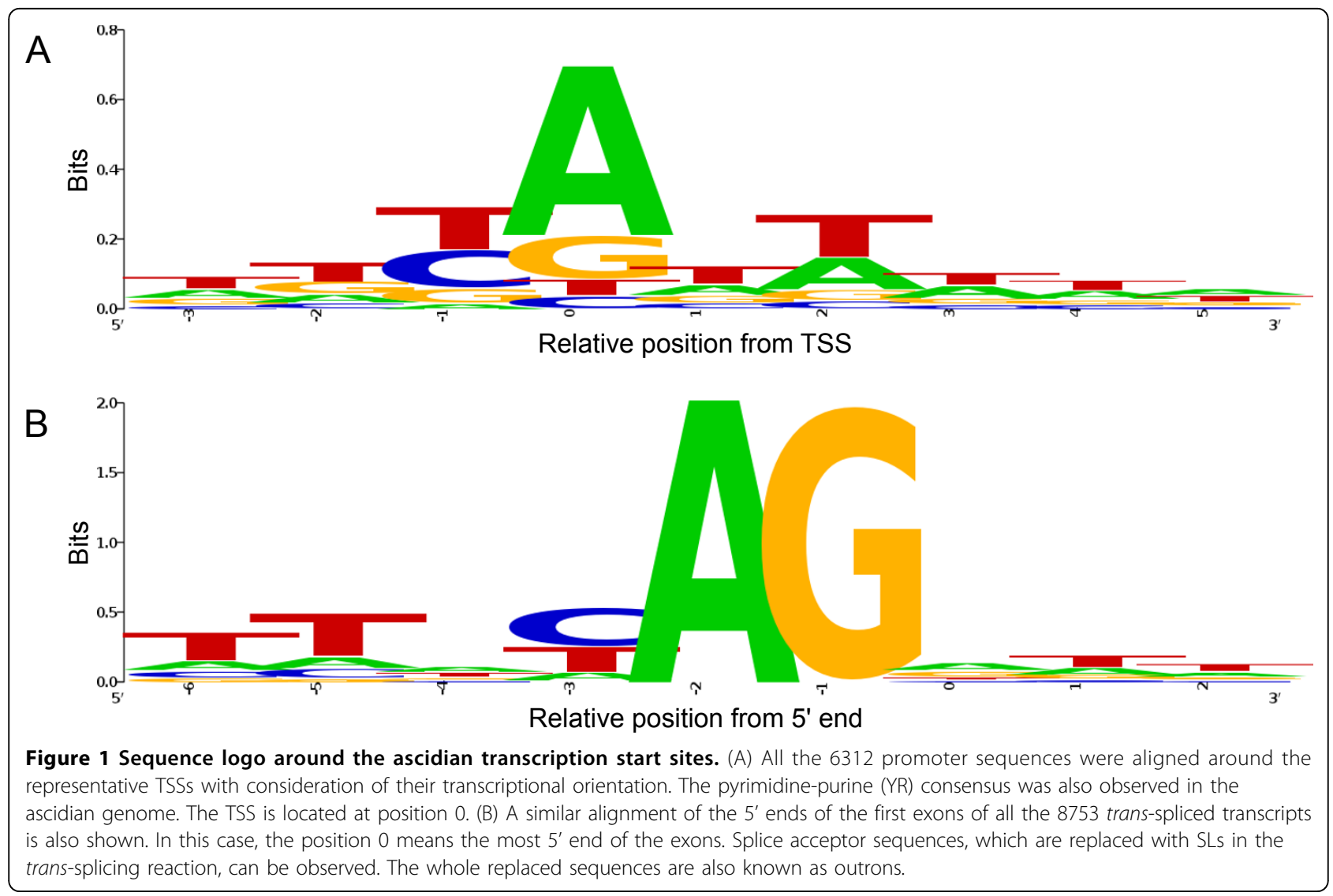




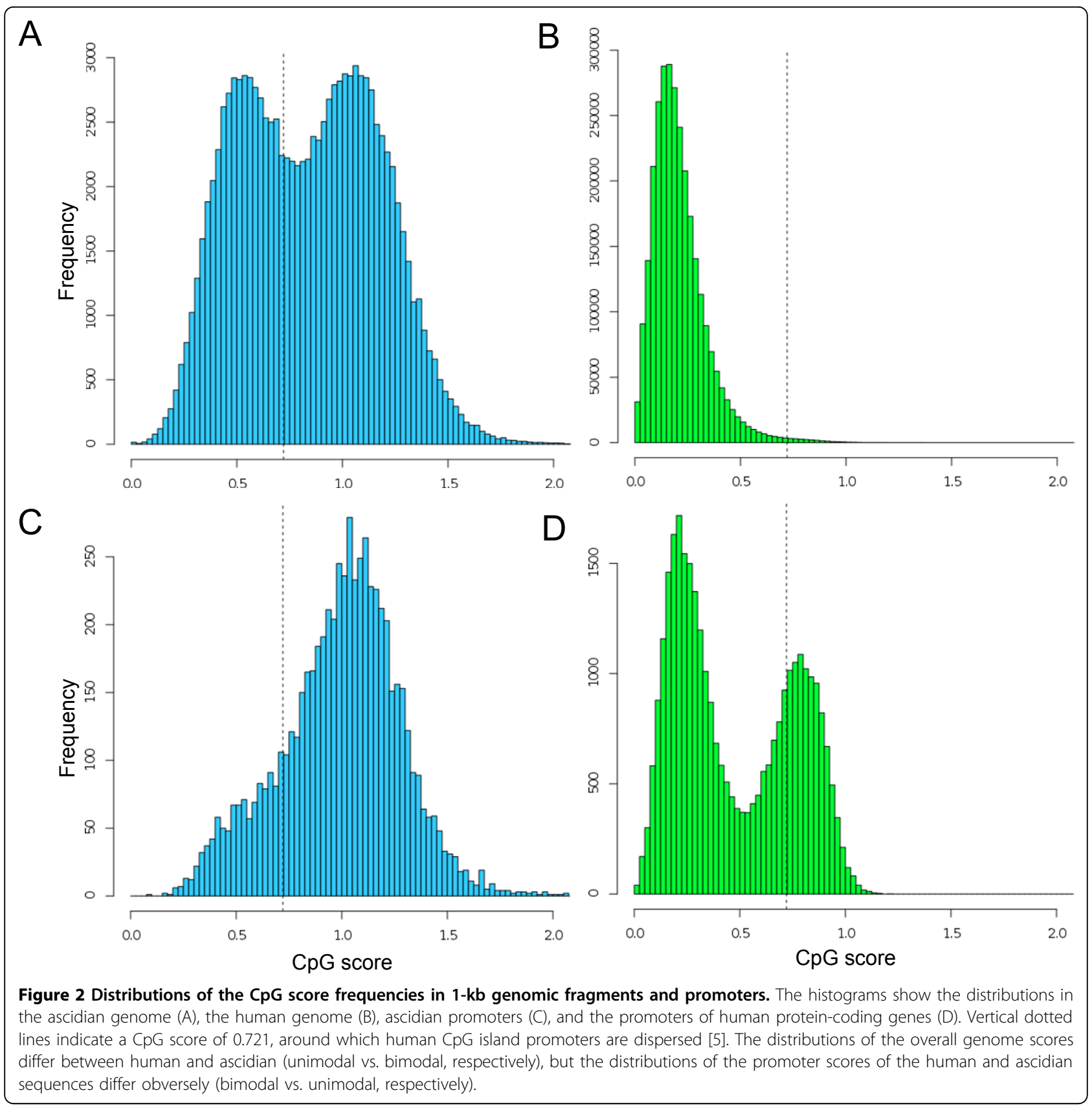

Lastly, we examined the usage of the four YR dinucleotides (CpA, CpG, TpA, and TpG) at the YR-consensus sites (positions -1 and 0 ). This analysis was performed using representative TSSs, which have a oneto-one correspondence with promoters. As noted above, these dinucleotides are preferentially used as TSSs in a wide range of animals [29]. However, the frequencies of the dinucleotides are not equivalent (Figure 5). CpA is the most commonly observed as the representative TSS in both ascidian and human genomes. The second preference is for $\mathrm{CpG}$ in human $\mathrm{CpG}$ island promoters.
The usages of $\mathrm{CpG}$ are $4.2 \%, 3.5 \%$, and $18.1 \%$ in the ascidian, human CpG-poor, and $\mathrm{CpG}$ island promoters, respectively. Although the ascidian promoters tended to exhibit high CpG scores (Figure 2), CpG seems to be used rarely as the transcription initiation point.

\section{Discussion}

The CpG island promoters seen in vertebrates are believed to have emerged from the deamination of other regions [17]. Therefore, it is plausible that the appearance of the two classes of vertebrate promoters is also a 
consequence of deamination, following the global DNA methylation that occurred early in vertebrate evolution $[16,30]$. Specific sequence motifs that function as transcription factor binding sites might have retained some CpG-rich sequences from the methylation and mutation to form CpG island promoters [31-33]. To confirm this hypothesis, we used a large-scale experimental approach to identify the TSSs of $C$. intestinalis. On the basis of our TSS information, we then examined the ascidian promoter sequences. The fact that the $\mathrm{CpG}$ scores, i.e. the ratios of the observed $\mathrm{CpG}$ number to the expected CpG number, tended to be quite high in the vicinity of the ascidian TSSs led us speculate CpG island promoters [16]. However, it had to be noted that the $\mathrm{G}+\mathrm{C}$ and CpG contents are low. When we applied the most conventional and conservative $\mathrm{CpG}$ island definition [8] to the promoters, only $3.5 \%$ (223 out of the 6313 promoters) meet the criteria. This is attributable to the fact that the ascidian $\mathrm{G}+\mathrm{C}$ content, approximately 0.36 , is much lower than the $\mathrm{G}+\mathrm{C}$ criterion of 0.5 (Figure $3 \mathrm{~B}$ ). Even at TSSs, the average ascidian $\mathrm{G}+\mathrm{C}$ content is approximately 0.4 at the most. Besides, the ascidian CpG score is much higher than the criterion of 0.6 (Figure $3 \mathrm{~A}$ ). If we try to define new criteria for the ascidian genome, the difference in the values for the TSSs and background sequences is much smaller than that observed for the human genome. The unique feature of the non-vertebrate deuterostome genomes, i.e. the presence of comparable amounts of CpG-poor and CpGrich domains [7], also hinders us in defining CpG islands in these animals.

Contrary to our initial expectation, we failed to identify $\mathrm{CPG}$ island-like promoters in the invertebrate genome. Instead, we found that the general features of ascidian promoters are similar to those of CpG-poor vertebrate promoters rather than to $\mathrm{CpG}$ island promoters. It is reasonable to consider CpG-poor promoters more ancient because they are found in a wide variety of eukaryotes [29]. Conversely, CpG island promoters must have appeared in an early stage of vertebrate evolution, derived by some mechanism, and have been adopted as important cis regulatory elements in descendant species. Because the CpG score is just the ratio of the observed to the expected numbers of dinucleotides, a high score does not necessarily mean a high frequency. We defined and used "CpG content", which showed a substantially different feature from $\mathrm{CpG}$ score in the ascidian genome (Figure $3 \mathrm{C}$ ). Note that the $\mathrm{CpG}$ score and $\mathrm{CpG}$ content profiles are dissimilar and similar in the ascidian and human genomes, respectively. The CpG content will also be important to scrutinize genomes especially of various animals other than mammals. It is unlikely that the conventional CpG island definitions using only $\mathrm{CpG}$ score, $\mathrm{G}+\mathrm{C}$ content, and length
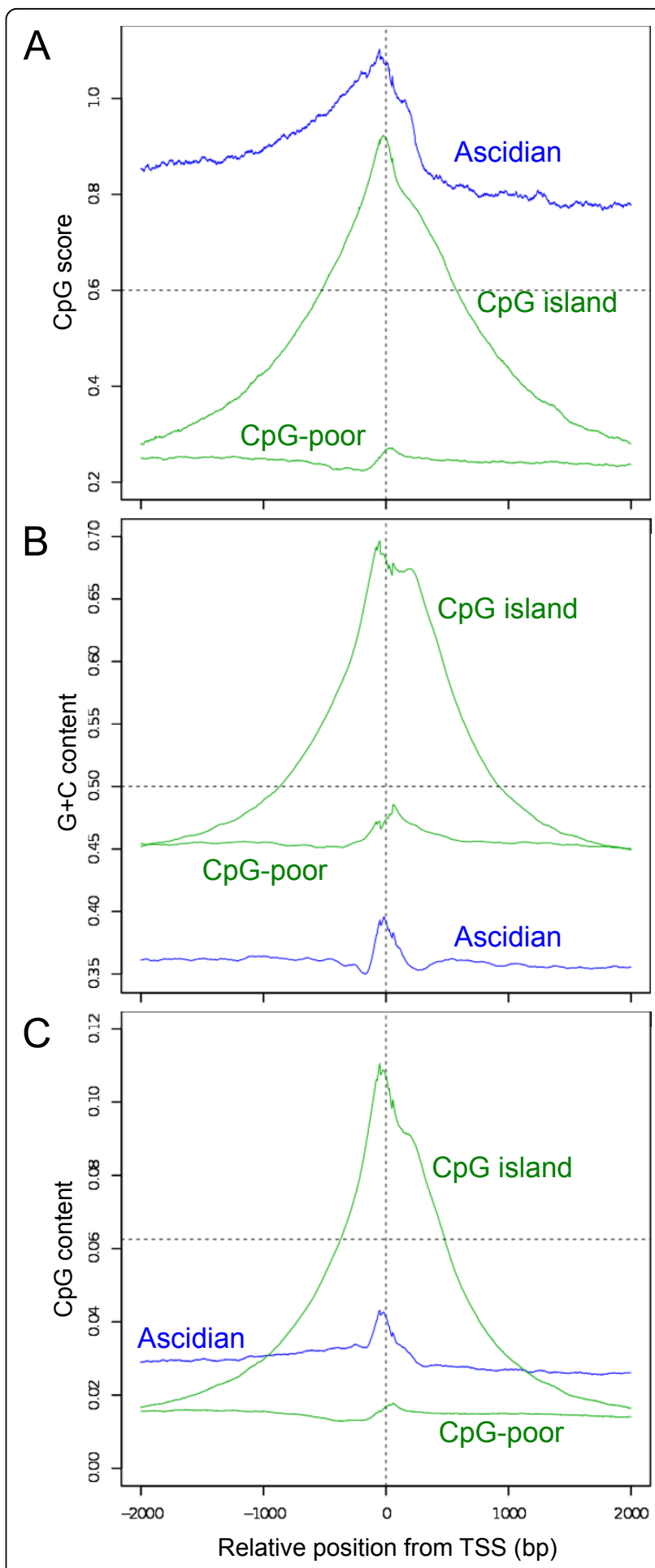

Figure 3 Changes in the $\mathrm{CpG}$ scores (A), G+C contents (B), and CpG contents around the TSS. The local $\mathrm{CpG}$ score, $\mathrm{G}+\mathrm{C}$ content, and $\mathrm{CpG}$ content at each position within a 4-kb region, with a moving window size of 100 bp, were averaged for the ascidian, human CpGpoor, and CpG island promoters. The horizontal axes indicate the position relative to the representative TSS. Horizontal dotted lines indicate the conventional criteria for vertebrate $\mathrm{CpG}$ islands (A, B) and the expected content of each dinucleotide, 1/16 (C). 
function in invertebrate genomes. Because the deamination of methylated $\mathrm{CpG}$ sites cannot explain the substantial increase in the $C p G$ and $G+C$ contents in the vicinity of vertebrate TSSs, we must search for and examine active mechanisms that may have given rise to CpG islands. The biased gene conversion [18,34], the condensation of CpG-rich protein-coding sequences by retrotransposition [35], and the expansion of elements containing the CpG dinucleotide [36] are potential molecular mechanisms. The fact that $\mathrm{CpG}$ islands are not conserved satisfactory among species [8] may indicate that $\mathrm{CpG}$ island loss and gain are active phenomena, occurring up to the present time, even in extant vertebrates.

The number of $C$. intestinalis genes is reported to be 15,254 in the $\mathrm{KH}$ gene model [25]. Whereas series of operonic genes have single promoters, alternative promoters have been reported for a large number of genes. The number of all RNA polymerase II promoters, including those of non-coding transcripts, may exceed 20,000. This study targeted the promoters that are active in the embryos. Although we believe that the 6312 promoters analysed here may well represent most of them, we eagerly await techniques with which to identify the TSSs of trans-spliced genes. Utilizing our data, the TSS of the TnI gene was recently identified as the first case for Ciona trans-spliced genes [36]. CpG island promoters cannot be seen at least for this gene.

\section{Conclusions}

We have experimentally identified and characterized ascidian promoter sequences as the primordial type of vertebrate promoter. As far as we know, this is the first case for non-vertebrate deuterostomes. The sequences near TSSs tend to exhibit high CpG score and high G $+C$ content, but their level and extent are actually restricted. Furthermore, the promoter sequences seem to be at least partially methylated. It is unlikely that they were the original type of vertebrate $\mathrm{CpG}$ island promoters. Rather than global methylation and subsequent deamination, some active mechanisms and maintaining mechanisms have presumably been required to form such a long and $\mathrm{CpG}$-condensed region in vertebrate animals.

The genomes of more than 50 vertebrate species have been sequenced and even more genomes will be sequenced in the future [38]. Now that an ascidian genome has been shown to lack CpG islands that function in promoter sequences, our curiosity is directed to primitive vertebrates, such as agnathans. It could be superficial to make a strong conclusion at this point. The searching for primitive organisms with $\mathrm{CpG}$ island promoters in order to determine the origin of $\mathrm{CpG}$ islands will certainly extend our understanding of the sophisticated roles of DNA methylation in higher eukaryotes [39-41].

\section{Methods \\ RNA extraction, oligo capping, and RNA-seq with the Illumina Genome Analyzer}

More than $200 \mu \mathrm{g}$ of total RNA was isolated from whole mid-tailbud-stage ascidian embryos (12-hour-old embryos), using ISOGEN (Nippon Gene) according to the manufacturer's protocol. The RNA was subjected to oligo-capping method [19]. In short, after successive treatments with bacterial alkaline phosphatase (TaKaRa) and tobacco acid pyrophosphatase (Ambion), the treated RNA was ligated to an RNA oligonucleotide with the sequence 5' - AAU GAU ACG GCG ACC ACC GAG AUC UAC ACU CUU UCC CUA CAC GAC GCU CUU CCG AUC UGG -3' using T4 RNA ligase (TaKaRa). After treatment with DNase I, the poly $(\mathrm{A})^{+}$ RNA was selected and used as the template for the firststrand cDNA synthesis with the primer 5'- CAA GCA GAA GAC GGC ATA CGA NNN NNN C -3'. The cDNA was then used as the template for PCR with the primers 5'- AAT GAT ACG GCG ACC ACC GAG -3' and 5'- CAA GCA GAA GAC GGC ATA CGA -3'. The products were size fractionated by polyacrylamide gel electrophoresis. Approximately $1 \mathrm{ng}$ of the 150-200-bp fraction was used for the sequencing reactions on the Illumina Genome Analyzer (Solexa). Both 36-cycle and 48-cycle sequencing reactions were performed on the same samples. The DNA sequences have been deposited in [DDBJ Sequence Read Archive: DRA000156].

\section{Sequence data analysis}

Illumina Pipeline (GAPipeline 1.0) was used to extract the sequenced reads from the image data. The spliced leaders (SLs) in the trans-spliced sequences were replaced with splice acceptor sequence "ag" for the subsequent mapping. The sequences were aligned to the $\mathrm{KH}$ assembly [25] using SeqMap [42] for the 36-cycle reads, or to BLAT [43] for the 48-cycle reads, because of the high rate of cis-splicing. Because of the highly polymorphic genic features of this organism [44], we used a $90 \%$ match criterion, including insertions and deletions. If the 5 ' end of a read was not aligned to the genome, the read was eliminated from the analysis. Multiple hits were removed, and only single best hits were considered for the subsequent analyses. Sequence logos were drawn with WebLogo 2.8.2 (http://weblogo.berkeley.edu/). The $\mathrm{CpG}$ score was defined as $C p G * N / C$ / $G$ with $C, G, C p G$, and $N$ observed numbers of $C, G$, and $C p G$ and the fixed window size, respectively. The CpG content defined in the present study was $C p G$ / ( N - 1). The assembly used for the human genome was UCSC hg18. To select human CpG-poor (CpG score < 


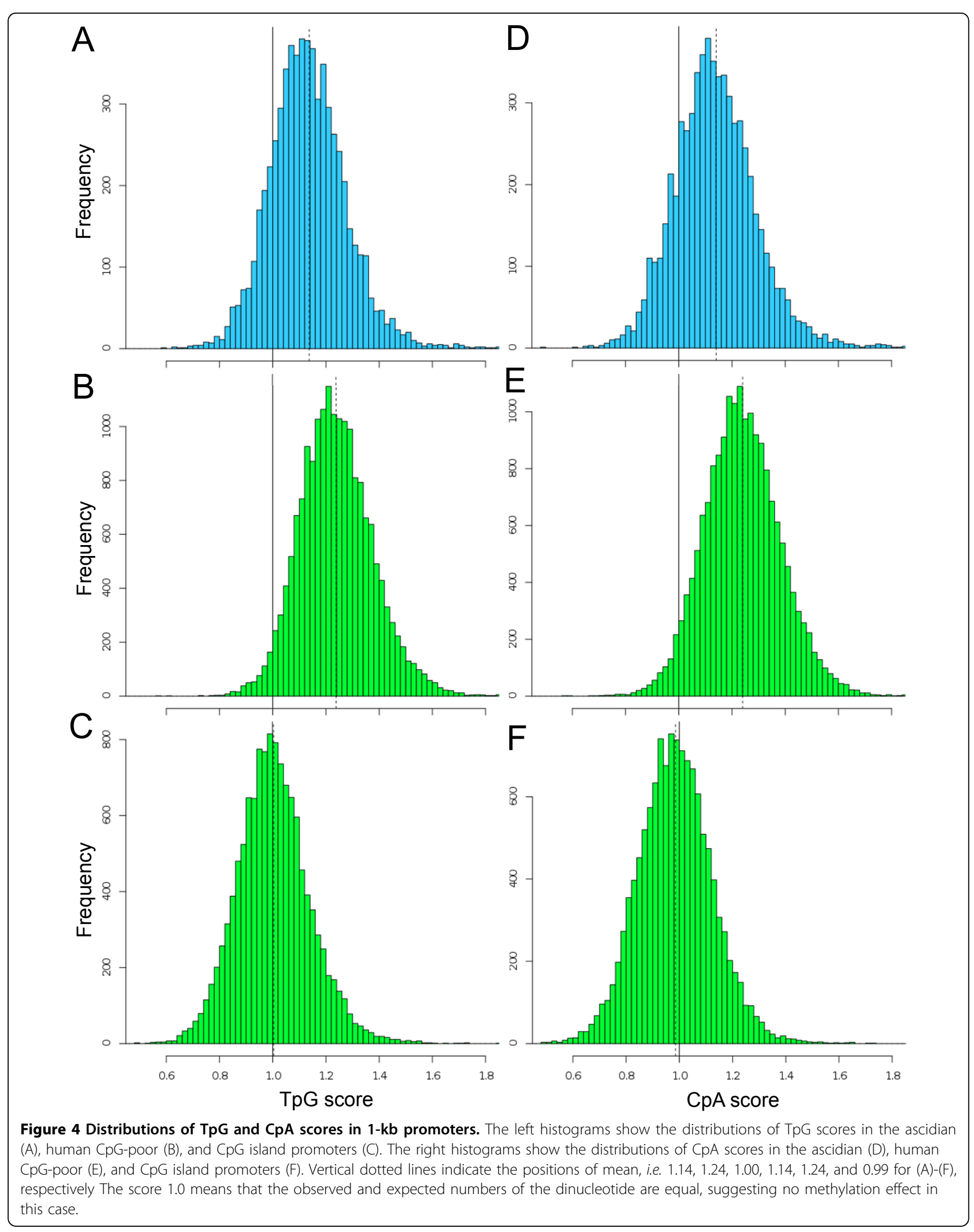



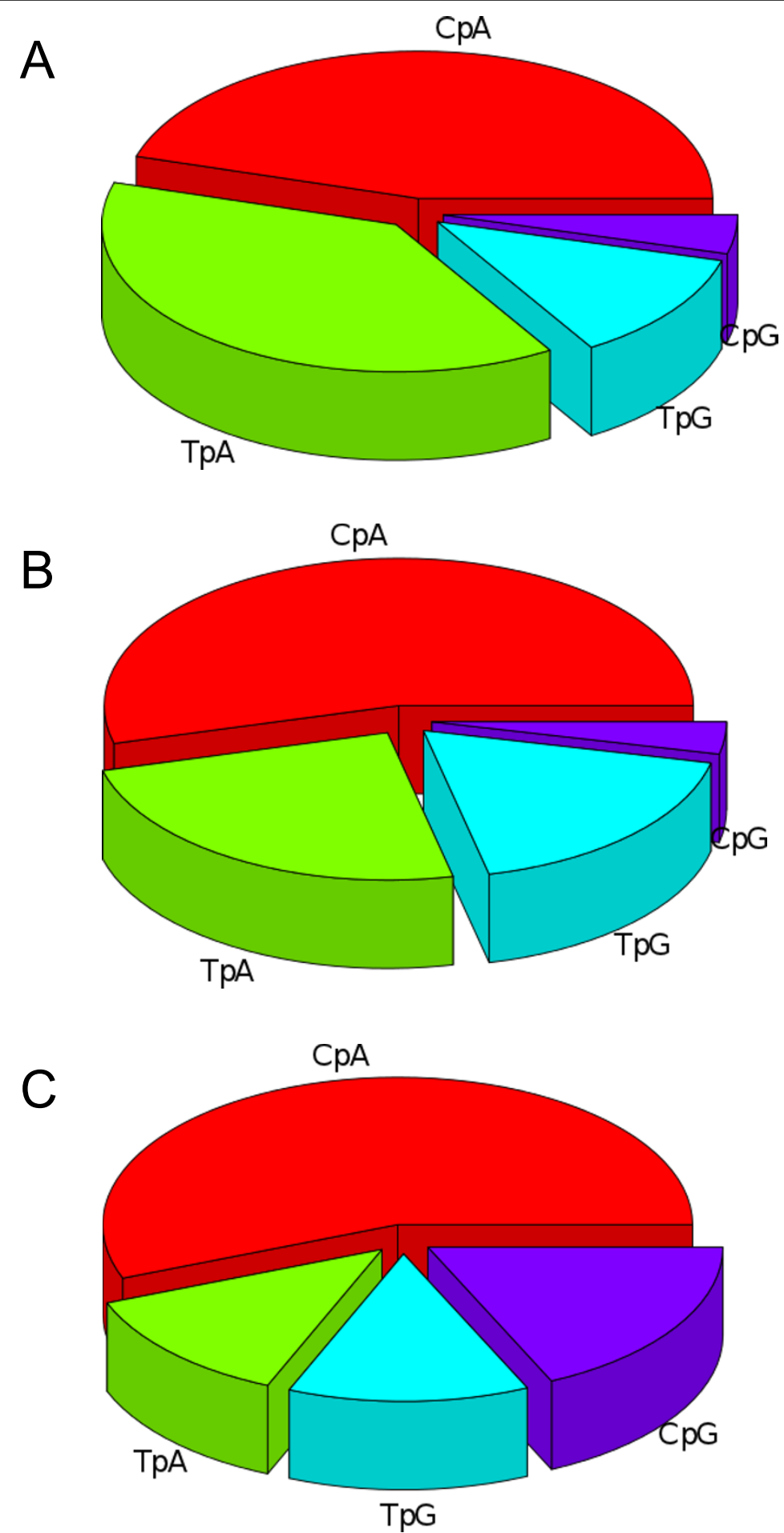

Figure 5 Usage of the four YR dinucleotides at the TSS. The proportional use of each YR dinucleotide at the representative TSSS was calculated for the three promoter groups: ascidian (A), human CpG-poor (B), and human CpG island promoters (C). Dinucleotides other than $\mathrm{CpA}, \mathrm{CpG}, \mathrm{TpA}$, and TpG were ignored in this analysis. Whereas $\mathrm{CpG}$ is the second most often used dinucleotide in human CpG-rich promoters, the dinucleotide is used least in ascidian and human CpG-poor promoters. 
$0.5)$ and $\mathrm{CpG}$-rich promoters ( $\mathrm{CpG}$ score $>0.6)$, we used DBTSS 6.0 (http://dbtss.hgc.jp/) and calculated CpG scores in 200-bp regions around representative TSSs [45]. The analysis was limited to protein-coding genes, but all the alternative promoters deposited in the database were included (out of all 101,436 promoters, 32,122 were for protein-coding genes). The numbers of CpG-poor and CpG-rich promoters were 18,034 and 12,493 , respectively. Dinucleotides other than pyrimidine-purine (YR) were not considered in the analysis of the usage of the YR motif. The total numbers of YR motifs at TSSs were $3,610,8,162$, and 8,610 for ascidian, human CpG-poor, and human CpG island promoters, respectively. All the sequence analyses were performed with Perl scripts, which are available upon request.

\section{Additional material}

\section{Additional file 1: Ciona intestinalis embryos at mid-tailbud stage}

(Figure S1) A photo of two C. intestinalis embryos at mid-tailbud stage, taken 12 hours after fertilization.

Additional file 2: Summary of the TSS-seq experiments (Tables S1 and S2) Quantitative data for the 6312 non-trans-spliced and 8753 transspliced transcripts which were annotated by the nearest genes.

Additional file 3: Changes in dinucleotide scores in the vicinity of TSSs (Figure S2) The score changes are shown for ascidian promoters (A), human CpG-poor promoters (B), and human CpG-rich promoters (C).

\section{List of abbreviations used}

TSS: transcription start site; SL: spliced leader

\section{Acknowledgements and funding}

The authors are grateful to Dr. Kenneth E. M. Hastings (McGill University) for discussions. We thank Ms. Ritsuko Kato and Ms. Mayu Fushimi (Ochanomizu University) for technical assistance. Computation time was provided by the supercomputer system at the Human Genome Centre, Institute of Medical Science, University of Tokyo. This work was supported by the Japan Society for the Promotion of Science (JSPS) through its "Funding Program for WorldLeading Innovative R\&D on Science and Technology (FIRST Program)", the Institute for Bioinformatics Research and Development (BIRD), Japan Science and Technology Agency (JST), KAKENHI (22310120 and 23770273), and the Global COE Program (Center of Education and Research for Advanced Genome-Based Medicine), MEXT, Japan.

This article has been published as part of BMC Genomics Volume 12 Supplement 3, 2011: Tenth International Conference on Bioinformatics - First ISCB Asia Joint Conference 2011 (InCoB/ISCB-Asia 2011): Computational Biology. The full contents of the supplement are available online at http:// www.biomedcentral.com/1471-2164/12?issue=S3.

\section{Author details}

'Human Genome Centre, Institute of Medical Science, University of Tokyo, Tokyo, Japan. ${ }^{2}$ Centre for Informational Biology, Ochanomizu University, Tokyo, Japan. 'Department of Life Science, Graduate School of Life Science, University of Hyogo, Hyogo, Japan. ${ }^{4}$ Department of Biology, Faculty of Science and Engineering, Konan University, Kobe, Japan. ${ }^{5}$ Department of Medical Genome Sciences, Graduate School of Frontier Sciences, University of Tokyo, Kashiwa, Japan.

\section{Authors' contributions}

K.O. conceived of the study, designed the study, and drafted the manuscript; R.Y. helped to analyze data; N.T, K.N., and T.G.K. prepared Ciona embryos and extracted RNA; Y.S. performed sequencing; and K.N. participated in the coordination of the study.

\section{Competing interests}

The authors declare that they have no competing interests.

Published: 30 November 2011

\section{References}

1. Ponger L, Li W: Evolutionary diversification of DNA methyltransferases in eukaryotic genomes. Mol Biol Evol 2005, 22:1119-1128.

2. Coulondre C, Miller JH, Farabaugh PJ, Gilbert W: Molecular basis of base substitution hotspots in Escherichia coli. Nature 1978, 274:775-780.

3. Zemach A, Zilberman D: Evolution of eukaryotic DNA methylation and the pursuit of safer sex. Curr Biol 2010, 20:R780-R785.

4. Simmen MW: Genome-scale relationships between cytosine methylation and dinucleotide abundances in animals. Genomics 2008, 92:33-40.

5. Okamura K, Matsumoto KA, Nakai K: Gradual transition from mosaic to global DNA methylation patterns during deuterostome evolution. BMC Bioinformatics 2010, 11(Suppl 7):S2.

6. Simmen MW, et al: Nonmethylated transposable elements and methylated genes in a chordate genome. Science 1999, 283:1164-1167.

7. Suzuki MM, Kerr ARW, De Sousa D, Bird A: CpG methylation is targeted to transcription units in an invertebrate genome. Genome Res 2007, 17:625-631.

8. Gardiner-Garden M, Frommer M: $\mathrm{CpG}$ islands in vertebrate genomes. J Mol Biol 1987, 196:261-282.

9. Bird AP: $\mathrm{CpG}$ islands as gene markers in the vertebrate nucleus. Trends in Genetics 1987, 3:342-347.

10. Bird A: DNA methylation patterns and epigenetic memory. Genes Dev 2002, 16:6-21

11. loshikhes IP, Zhang MQ: Large-scale human promoter mapping using CpG islands. Nat Genet 2000, 26:61-63.

12. Yamashita R, Suzuki Y, Sugano S, Nakai K: Genome-wide analysis reveals strong correlation between $\mathrm{CpG}$ islands with nearby transcription start sites of genes and their tissue specificity. Gene 2005, 350:129-136.

13. Saxonov S, Berg P, Brutlag DL: A genome-wide analysis of CpG dinucleotides in the human genome distinguishes two distinct classes of promoters. Proc Natl Acad Sci USA 2006, 103:1412-1417.

14. Weber $M$, et al: Distribution, silencing potential and evolutionary impact of promoter DNA methylation in the human genome. Nat Genet 2007, 39:457-466.

15. Kasahara $M$, et al: The medaka draft genome and insights into vertebrate genome evolution. Nature 2007, 447:714-719.

16. Elango $\mathrm{N}, \mathrm{Yi}$ SV: DNA methylation and structural and functional bimodality of vertebrate promoters. Mol Biol Evol 2008, 25:1602-1608.

17. Bird AP: CpG-rich islands and the function of DNA methylation. Nature 1986, 321:209-213.

18. Cohen NM, Kenigsberg E, Tanay A: Primate CpG islands are maintained by heterogeneous evolutionary regimes involving minimal selection. Cell 2011, 145:773-786

19. Maruyama K, Sugano S: Oligo-capping: a simple method to replace the cap structure of eukaryotic mRNAs with oligoribonucleotides. Gene 1994, 138:171-174.

20. Tsuchihara $\mathrm{K}$, et al: Massive transcriptional start site analysis of human genes in hypoxia cells. Nucleic Acids Res 2009, 37:2249-2263.

21. Delsuc F, Brinkmann H, Chourrout D, Philippe H: Tunicates and not cephalochordates are the closest living relatives of vertebrates. Nature 2006, 439:965-968.

22. Vandenberghe $A E$, Meedel $T H$, Hastings KE: mRNA 5'-leader trans-splicing in the chordates. Genes Dev 2001, 15:294-303.

23. Hastings KE: SL trans-splicing: easy come or easy go? Trends Genet 2005, 21:240-247.

24. Satou Y, et al: Genomic overview of mRNA 5 '-leader trans-splicing in the ascidian Ciona intestinalis. Nucleic Acids Res 2006, 34:3378-3388.

25. Satou $Y$, et al: Improved genome assembly and evidence-based global gene model set for the chordate Ciona intestinalis: new insight into intron and operon populations. Genome Biol 2008, 9:R152.

26. Suzuki $Y$, et al: Diverse transcriptional initiation revealed by fine, largescale mapping of mRNA start sites. EMBO Rep 2001, 2:388-393. 
27. Yamashita R, Wakaguri H, Sugano S, Suzuki Y, Nakai K: DBTSS provides a tissue specific dynamic view of Transcription Start Sites. Nucleic Acids Res 2010, 38:D98-D104.

28. FitzGerald PC, Sturgill D, Shyakhtenko A, Oliver B, Vinson C: Comparative genomics of Drosophila and human core promoters. Genome Biol 2006, 7:R53.

29. Juven-Gershon T, et al: The RNA polymerase II core promoter - the gateway to transcription. Curr Opin Cell Biol 2008, 20:253-259.

30. Tweedie S, Charlton J, Clark V, Bird A: Methylation of genomes and genes at the invertebrate-vertebrate boundary. Mol Cell Biol 1997, 17:1469-1475.

31. Macleod D, Charlton J, Mullins J, Bird AP: Sp1 sites in the mouse aprt gene promoter are required to prevent methylation of the $\mathrm{CpG}$ island. Genes Dev 1994, 8:2282-2292.

32. Brandeis $\mathrm{M}$, et al: $\mathrm{Sp} 1$ elements protect a $\mathrm{CpG}$ island from de novo methylation. Nature 1994, 371:435-438.

33. Straussman, et al: Developmental programming of $\mathrm{CpG}$ island methylation profiles in the human genome. Nat Struct Mol Biol 2009, 16:564-571.

34. Duret $\mathrm{L}$, Galtier N: Biased gene conversion and evolution of mammalian genomic landscapes. Annu Rev Genomics Hum Genet 2009, 10:285-311.

35. Okamura K, Nakai K: Retrotransposition as a source of new promoters. Mol Biol Evol 2008, 25:1231-1238.

36. Okamura K, Wintle RF, Scherer SW: Characterization of the differentially methylated region of the Impact gene that exhibits Glires-specific imprinting. Genome Biol 2008, 9:R160

37. Khare $P$, et al: Cross-validated methods for promoter/transcription start site mapping in SL trans-spliced genes, established using the Ciona intestinalis troponin I gene. Nucleic Acids Res 2011, 39:2638-2648.

38. Haussler, et al: Genome 10K: a proposal to obtain whole-genome sequence for 10,000 vertebrate species. J Hered 2009, 100:659-674.

39. Egger $G$, Liang $G$, Aparicio A, Jones PA: Epigenetics in human disease and prospects for epigenetic therapy. Nature 2004, 429:457-463.

40. Reik W: Stability and flexibility of epigenetic gene regulation in mammalian development. Nature 2007, 447:425-432.

41. Law JA, Jacobsen SE: Establishing, maintaining and modifying DNA methylation patterns in plants and animals. Nat Rev Genet 2010, 11:204-220.

42. Jiang $H$, Wong WH: SeqMap: mapping massive amount of oligonucleotides to the genome. Bioinformatics 2008, 24:2395-2396.

43. Kent WJ: BLAT-the BLAST-like alignment tool. Genome Res 2002, 12:656-664.

44. Dehal $P$, et al: The draft genome of Ciona intestinalis: insights into chordate and vertebrate origins. Science 2002, 298:2157-2167.

45. Yamashita R, et al: DBTSS: DataBase of Human Transcription Start Sites, progress report 2006. Nucleic Acids Res 2006, 34:D86-D89.

doi:10.1186/1471-2164-12-S3-S7

Cite this article as: Okamura et al.: Profiling ascidian promoters as the primordial type of vertebrate promoter. BMC Genomics 2011 12(Suppl 3): S7.

\section{Submit your next manuscript to BioMed Central and take full advantage of:}

- Convenient online submission

- Thorough peer review

- No space constraints or color figure charges

- Immediate publication on acceptance

- Inclusion in PubMed, CAS, Scopus and Google Scholar

- Research which is freely available for redistribution 